Revue internationale P.M.E.

Économie et gestion de la petite et moyenne entreprise

Revue

internationale

PME

\title{
La modernité chez le tontineur à Niamey
}

\section{Christian E. Rietsch}

Volume 5, numéro 3-4, 1992

URI : https://id.erudit.org/iderudit/1008157ar

DOI : https://doi.org/10.7202/1008157ar

Aller au sommaire du numéro

Éditeur(s)

Presses de l’Université du Québec

ISSN

0776-5436 (imprimé)

1918-9699 (numérique)

Découvrir la revue

Citer cet article

Rietsch, C. E. (1992). La modernité chez le tontineur à Niamey. Revue internationale P.M.E., 5(3-4), 117-139. https://doi.org/10.7202/1008157ar

\section{Résumé de l'article}

Les tontines passent fréquemment pour une forme financière résiduelle et vouée à la disparition. Notre enquête nous prouve, au contraire, qu'à Niamey une large fraction de la population adhère aux tontines et ceci en particulier parmi les adultes jeunes et actifs.

De plus, les participants aux tontines sont des individus aux revenus plus élevés et aux sources de revenus plus modernes que les autres ; généralement, ils ont accès au monde financier formel, mais ne négligent pas pour autant les modalités financières plus traditionnelles.

Finalement, la tontine apparaît comme un actif financier parmi d'autres et le tontineur comme un homme moderne qui pratique une gestion diversifiée d'actif financier en connaissant les avantages et les inconvénients de chacun.
Ce document est protégé par la loi sur le droit d'auteur. L’utilisation des services d’Érudit (y compris la reproduction) est assujettie à sa politique d'utilisation que vous pouvez consulter en ligne.

https://apropos.erudit.org/fr/usagers/politique-dutilisation/ 


\title{
La modernité chez le tontineur à Niamey
}

\author{
Christian E. RIETSCH* \\ Université d'Orléans \\ Université de Niamey
}

\begin{abstract}
RÉSUMÉ
Les tontines passent fréquemment pour une forme financière résiduelle et vouée à la disparition. Notre enquête nous prouve, au contraire, qu'à Niamey une large fraction de la population adhere aux tontines et ceci en particulier parmi les adultes jeunes et actifs.

De plus, les participants aux tontines sont des individus aux revenus plus élevés et aux sources de revenus plus modernes que les autres; généralement, ils ont accès au monde financier formel, mais ne négligent pas pour autant les modalités financières plus traditionnelles.

Finalement, la tontine apparaît comme un actif financier parmi d'autres et le tontineur comme un homme moderne qui pratique une gestion diversifiée d'actif financier en connaissant les avantages et les inconvénients de chacun.
\end{abstract}

\section{SUMMARY}

Tontines often pass as outdated and residual financial instruments which are bound to disappear soon. However this study shows that a large proportion of the population in Niamey uses them, particularly young working adults.

Their participants enjoy more-than-average income, have access to more modern sources of income than the majority and yet do not neglect traditional systems of finance. Thus tontines appear as one financial asset among others and participants as operators who are eager to diversify their portfolio after careful consideration of each instrument's advantages and drawbacks.

* Christian Rietsch a fait ses études à l'Université de Strasbourg. Docteur ès sciences économiques en 1981 à Nantes, maître de conférences à l'Université d'Orléans en 1985. Il travaille dans le cadre de la coopération française, d'abord comme fiscaliste au Zaïre, puis comme enseignant à l'Université de Bangui (République centrafricaine). Il est actuellement détaché à l'Université de Niamey (Niger). S'intéresse aux problèmes de développement, d'ajustement structurel et de finances informelles. Adresse : B.P. 12442 Niamey, Niger ou s/c M. Bruno Ponson, ESCP, 79, avenue de la République, 75443 Paris Cedex 11, France. 


\section{RESUMEN}

Las tontinas son a menudo consideradas como una forma financiera residual y en vías de desaperición.

Nuestra encuesta nos demuestra sin embargo que en Niamey una amplia franja de la poblacion se suscribe a las tontinas, en particular los jóvenes activos.

Además, los suscriptores de tontinas son individuos con rentas más elevadas y con fuentes de ingresos mas modernas que los otros. Por lo general tienen acceso al mundo financiero formal, sin descuidar por ello las modalidades financieras tradicionales.

Por último, la tontina aparece como un activo financiero entre otros, y el suscriptor de tontinas como un hombre moderno que practica una gestión diversificada de activos financieros y que conoce sus venjatas e inconvenientes.

\section{Introduction}

Parmi les économistes, il existe un très large consensus pour admettre le rôle crucial de l'épargne dans la croissance des pays, et ceci indépendamment de leurs cadres idéologiques ou doctrinaux : l'épargne apparaît comme un facteur essentiel du développement, en ce sens que transformée aujourd'hui en investissements, elle est déterminante du revenu de demain.

Or, l'un des diagnostics les plus courants de la crise qui frappe les économies africaines est la faiblesse de l'épargne. Les sources extérieures d'épargne étant réduites, les structures financières modernes, en difficulté, on a cherché s'il existait des gisements nationaux d'épargne qui n'avaient pas encore été prospectés. C'est dans ces conditions que l'on s'est souvenu que l'épargne qui passe par les institutions financières formelles ne constitue qu'une fraction de l'épargne totale, mais qu'une autre fraction transite par les individus qui s'organisent eux-mêmes pour faire face à l'avenir. On a alors recherché, pour les mobiliser (éventuellement en les faisant transiter par le système financier formel) les ressources financières informelles. De grands espoirs ont alors été mis dans la mobilisation de l'épargne informelle ${ }^{1}$. C'est dans ce cadre que dans de nombreux pays des études ont été lancées pour préciser ces flux souterrains d'épargne et leurs affectations. Au Niger, en particulier, une des études pionnières qui ont permis d'établir les premières conclusions citées plus haut avait concerné les finances du monde rural. Il nous a semblé que la connaissance urbaine de ce phénomène manquait et que l'on ne

1. Par exemple, on lit dans le même rapport que « la circulation des liquidités dans les clubs indigènes [...] représente une base éventuelle extrêmement prometteuse pour la création d'une organisation coopérative plus formelle d'épargne et de prêts " (D. Grahma et alii, 1987). 
pouvait transposer à la ville de Niamey les comportements d'épargne notés dans les villages de la campagne sahélienne.

C'est pourquoi, avec le soutien de l'UREF (Université des réseaux d'expression française), une équipe de chercheurs du Département d'économie de la Faculté des sciences économiques et juridiques de l'Université de Niamey s'est constituée, en liaison avec l'Institut orléanais de finance, pour étudier ces phénomènes financiers informels et, en particulier, le plus connu et le plus visible d'entre eux, le phénomène tontinier.

Précisons rapidement ce que l'on entend par tontine.

Une tontine est une association de personnes se réunissant à intervalles donnés et versant chacune une cotisation dont le montant global est remis à tour de rôle à chaque membre.

Les individus qui appartiennent à l'association sont aussi appelés membres de la tontine, participants, tontineurs ou cotisants.

La cotisation que verse chaque membre de la tontine est parfois dénommée part ou versement.

Ramasser l'ensemble des cotisations du jour consiste à procéder à la levée de la tontine.

Supposons l'association formée de $n$ membres ( $n$ sera supérieur ou égal à 2, car tout seul il est difficile de former une association !) ; si au cours d'une réunion, chaque participant verse une somme $a$, alors, à un moment, sera rassemblée la somme totale mise en mouvement ce jour, soit na.

Un des membres de la tontine récupère cette somme ; on lui remet donc na ; cette somme est aussi appelée levée.

Quand chacun des $n$ membres de la tontine a levé une fois, on dit que la tontine a bouclé son cycle ou que l'ensemble des membres est désintéressé.

La somme globale mise en mouvement par l'ensemble des tontineurs au cours d'un cycle est évidemment égale à $n$ fois la somme mise en jeu lors d'une réunion, $n a$ : elle est donc égale à $n^{2} a$.

D'innombrables variantes sont possibles sur ces données de base, mais toutes reposent sur ces relations simples. Le schéma abstrait indiqué ici se greffe sur une réalité sociale très riche, d'où le foisonnement de problèmes concrets posés à la tontine et l'éventail des solutions apportées².

2. Pour avoir une idée tant de la réalité sociale que de la diversité des problèmes techniques dans la tontine, voir C. Rietsch, 1991. 


\section{Participation aux sociétés de tontine}

Le protocole ${ }^{3}$ de l'enquête sur les tontines en milieu urbain à Niamey permettait de générer deux sous-ensembles dans la population enquêtée :

- les enquêtés qui pratiquent la tontine ou tontineurs;

- les enquêtés non tontineurs susceptibles de servir de population-témoin.

Au dépouillement des 193 questionnaires, il apparaît que 58 enquêtés appartiennent à une tontine au moment de l'enquête. Cela nous donne un taux de participation de $30 \%$ à la pratique tontinière dans l'échantillon ${ }^{4}$. Ce taux est remarquablement élevé et dépasse quelque peu celui que l'on pouvait attendre d'après les indications fournies par d'autres enquêtes, non centrées sur la pratique de la tontine, mais qui avaient néanmoins posé la question de la participation.

Ainsi, l'Enquête budget-consommation du ministère du Plan et de la Statistique (non encore dépouillée) en laisse entrevoir moins de l'avis officieux des enquêteurs. De même les travaux généraux sur le financement du secteur informel (Oudin, 1989) à Niamey ou ceux plus spécifiques montrent que les tailleurs n'utilisent jamais la tontine pour financer leur développement (K. Liman Tinguiri, 1988).

Par contre, la connaissance par les membres de l'équipe des milieux locaux autorisait la prévision de tels pourcentages.

Les résultats $d u$ dépouillement montrent un taux de participation aux tontines très différente selon le sexe. En effet, le taux de participation aux tontines parmi les hommes est de $20,2 \%$ tandis que, parmi les femmes, il est de $41,6 \% 5$. Les résultats sont les suivants :

3. L'enquête, dont cette étude présente les premiers résultats, a été menée par une équipe de l'Université de Niamey, dirigée par K. Liman Tinguiri et C. Rietsch. Les fichiers informatiques ont été préparés par K. Liman Tinguiri et l'auteur a bénéficié de nombreuses observations de sa part; l'auteur reste cependant seul responsable de ce texte.

4. Compte tenu de la taille de l'échantillon, on peut calculer un intervalle de confiance pour la pratique de la tontine à Niamey parmi les adultes : ce taux de participation se situe avec $95 \%$ de chance dans l'intervalle $23,5 \%$ - 36,5\% avec une espérance mathématique de $30 \%$. Les intervalles de confiance donnés ici correspondent à des calculs effectués sur un tirage élémentaire au hasard et avec remise. Or, notre enquête a été effectuée à l'aide d'un tirage à plusieurs niveaux, les unités de chaque niveau étant sensiblement de même taille. La prise en compte exacte de la taille de chaque unité conduirait à un intervalle de confiance un peu plus large. 


\section{TABLEAU 1}

Différenciation dans la pratique de la tontine selon les sexes à Niamey

\begin{tabular}{lccc}
\hline $\begin{array}{l}\text { Sexe } \\
\text { Pratique la tontine }\end{array}$ & Masculin & Féminin & Total \\
\hline Oui & 21 & 37 & 58 \\
Non & 83 & 52 & 135 \\
Total & 104 & 89 & 193 \\
\hline
\end{tabular}

Un test du $\chi^{2}$ montre que cette différenciation entre les comportements masculins et féminins est hautement significative ${ }^{6}$. Les hommes sont légèrement plus nombreux que les femmes dans l'échantillon (104 contre 89). Mais les femmes tontinent nettement plus que les hommes (leur taux de participation est de $41,6 \%$ contre $20,2 \%$ ). Compte tenu du déséquilibre initial des effectifs selon les sexes, on constate que $63,8 \%$ des participants aux tontines sont des femmes et que $36,2 \%$ des tontineurs sont des hommes. Ainsi, à Niamey, le tontineur a de fortes chances d'être... une tontineuse!

\section{2. Éléments de différenciation dans la pratique de la tontine}

En dehors de la différenciation sexuelle, les autres éléments sociaux ne semblent pas très probants en tant que facteurs d'explication des particularités de comportement.

Par contre, les éléments économiques paraissent indiquer une forte différenciation et expliquer largement la pratique de la tontine.

5. Ce qui nous donne, pour la population féminine de Niamey et dans les conditions exposées ci-dessus, un intervalle de confiance à $95 \%$ compris entre $31,4 \%$ et $51,8 \%$.

6. $c^{2}$ significatif à 1 pour 1000 environ. 


\subsection{La faible différenciation sociale dans la pratique tontinière}

\subsubsection{Groupes ethniques et pratique tontinière}

Parmi les nationaux, nous avons sélectionné les 6 groupes ethniques principaux et un groupe « divers » regroupant les autres ethnies du pays.

La répartition des nationaux suit presque exactement la répartition entre tontineurs et non-tontineurs au point qu'il n'existe presque aucune différence entre les groupes ethniques quant à la pratique de la tontine. Aucun biais ethnique n'apparaît dans la pratique tontinière ou son absence.

Ce point est à comparer au cas camerounais où la situation est totalement opposée : le groupe ethnique Bamiléké, beaucoup plus que tous les autres, pratique la tontine.

Dans le cas nigérien, en ville, il ne semble pas que la pratique tontinière soit plus particulière à un groupe, au moins à Niamey. Notons que ceci réfute l'opinion courante qui attribue la pratique tontinière essentiellement au groupe haoussa ${ }^{7}$; même si ce groupe semble dans notre échantillon pratiquer légèrement plus la tontine que les autres groupes, les tests statistiques n'indiquent rien de significatif à cette différence très faible qui peut très bien s'expliquer par les fluctuations normales d'échantillonnage.

L'intégration des étrangers dans l'enquête n'en modifie pas la signification. Le déséquilibre observé entre les étrangers qui pratiquent la tontine et ceux qui ne la pratiquent pas n'est pas statistiquement significatif ${ }^{8}$. Quoique la faible participation aux tontines des étrangers ne soit pas statistiquement significative, il n'est pas exclu que certains d'entre eux se soient, plus que les nationaux, méfiés du questionnaire, de l'enquête ou de l'enquêteur et aient été réticents à avouer une pratique pouvant être considérée, à tort ou à raison, comme suspecte ou susceptible d'être pénalisée?.

7. Il semble en effet que dans le milieu rural le groupe haoussa soit particulièrement connu pour pratiquer la tontine. Voir dans ce sens : M.F. Diarra, 1984 ; G. Nicolas, H. Magagi, M. dan Mouche, 1968 ; D. Graham et alii, 1987.

8. Compte tenu de la faiblesse des effectifs théoriques parmi les étrangers pratiquant la tontine, une correction de Yates s'impose pour le calcul du $\mathrm{c}^{2}$; dans ces conditions, celui-ci ressort à 1,13 , largement inférieur au seuil de signification à $5 \%$, soit 3,84 . On peut donc énoncer avec beaucoup de sécurité l'absence de différence significative entre nationaux et étrangers quant à la participation aux tontines.

9. Les deux plus grosses tontines que nous connaissons à Niamey, dont l'une est gérée par un professionnel, sont exclusivement ou majoritairement béninoises ; leurs levées sont respectivement de 2,5 et 2 millions de FCFA, chiffre à comparer avec celui de notre échantillon (300 000 FCFA). 


\subsection{2. Âge et pratique de la tontine}

L'enquête concernait les adultes des deux sexes; était considéré comme adulte un individu ayant eu son vingt et unième anniversaire. Les classes d'âge étaient envisagées de façon assez large (de 10 ans en 10 ans) et regroupées au-delà de 50 ans ${ }^{10}$.

a) En ce qui concerne les hommes, la distribution obtenue indique les caractéristiques suivantes :

- la moyenne d'âge est de 36 ans environ pour les tontineurs et de 53 ans parmi les non-tontineurs ;

- toutefois, la différence de répartition par âge entre tontineurs et nontontineurs n'est pas statistiquement significative; le $\chi^{2}$ calculé est de 4,521 , alors qu'il ne serait significatif à $5 \%$ que s'il dépassait 7,81 ;

- dans la classe des 31 à 40 ans, la pratique tontinière est remarquablement peu répandue : il s'agit pourtant d'une tranche d'âge où l'épargne est habituellement considérée comme particulièrement nécessaire pour s'établir sérieusement ;

- à l'inverse, au-dessus de 50 ans, la pratique tontinière est particulièrement forte, tant relativement à l'effectif de la classe qu'à celui des tontineurs. Or fréquemment, on considère qu'il s'agit d'un âge de la vie où l'on n'épargne plus tellement parce que l'on est établi et que le terme de la vie s'approche (ceci d'autant plus en Afrique, où l'espérance de vie est plus courte).

Diverses hypothèses expliquant ce comportement peuvent être émises :

- les gens âgés ont depuis très longtemps une propension à épargner supérieure à celle des générations plus jeunes ;

- l'absence ou la modicité des retraites et pensions au Niger obligent les personnes les plus âgées à épargner intensément pour assurer leurs vieux jours, la confiance dans la solidarité intergénération devenant plus limitée ;

- les personnes les plus âgées, aux revenus les plus importants, gèrent ceux-ci à l'aide des tontines.

Pour apprécier la pertinence de ces hypothèses, il conviendra d'observer la nature des revenus de ces personnes.

10. Un large intervalle de classe n'est pas un handicap dans un pays où l'état civil est très imprécis pour les personnes âgées de plus de 30 ans. 
b) En ce qui concerne les femmes, on note que celles qui ont entre 31 à 40 ans constituent le seul groupe où celles qui pratiquent la tontine sont plus nombreuses que celles qui ne la pratiquent pas. La différence de comportement selon l'âge, parmi les femmes, est très significative puisque le $\chi^{2}$ calculé sur l'ensemble des chiffres du tableau ressort à $12,44^{11}$.

Une étude plus précise nous montre alors que :

- l'âge moyen des tontineuses et des non-tontineuses est quasiment le même : la différence ne dépasse pas 6 mois ;

- dans la classe des 31 à 40 ans, une caractéristique flagrante apparaît : c'est dans cette tranche d'âge que les femmes participent aux tontines. Les deux tiers d'entre elles le font, alors qu'elles sont moins du tiers en moyenne dans les autres classes.

Les hypothèses expliquant ce comportement peuvent être multiples : gestion de l'encaisse d'un petit commerce, gestion des dépenses du ménage et plus généralement, activité intense de la femme.

\subsubsection{Différenciation culturelle et pratique tontinière}

Les premiers dépouillements ont été effectués sur la pratique de la langue française et de l'arabe. Aucune différence significative n'apparaît dans les deux cas. Il est d'ailleurs fort possible que les pratiques des langues soient surévaluées par les intéressés. Il s'agit d'un phénomène bien connu dans de nombreuses enquêtes. Ce qui est plus important est l'absence de différence dans le comportement pour ce qui concerne la pratique d'une langue et la participation ou non à une tontine : cette constatation a de fortes chances d'être vraie, puisque la question de la participation aux tontines ne s'est posée que bien plus tard, évitant ainsi les biais de l'autosélection.

Les mêmes conclusions concernant la non-différenciation de la pratique tontinière selon l'usage d'une langue se retrouvent en ce qui concerne le diplôme obtenu. La question concernant les diplômes demandait quel était « le diplôme le plus élevé de l'individu ». Or, il est remarquable de constater que les distributions des diplômes sont quasi identiques que l'on participe à une tontine ou non. Il est probable, là aussi, que les individus ayant atteint un niveau sans obtenir un diplôme s'en soient attribués un dans leur réponse. En effet, parmi les adultes de Niamey, il est pour le moins étonnant de constater que $50 \%$ possèdent un diplôme ! Cette proportion est manifestement surévaluée d'après tout ce que nous savons par ailleurs.

11. À environ $0,5 \%$. 


\section{Conclusion}

Parmi l'ensemble des déterminants socioculturels, seuls deux éléments semblent jouer dans la pratique tontinière : le sexe et l'âge. En effet, les femmes tontinent nettement plus que les hommes et, parmi les femmes, celles entre 31 et 40 ans en font une pratique courante. Les autres variables socioculturelles étudiées ne sont pertinentes à aucun point de vue, qu'il s'agisse de la population masculine ou que, dans l'ensemble de la population, l'on tienne compte ou non de l'âge, de la pratique des langues arabes ou française ou encore du diplôme obtenu.

\subsection{La différenciation sur les variables économiques}

Autant les variables socioculturelles dépouillées ne semblent pas très éclairantes (excepté le sexe et l'âge pour les femmes) autant les variables économiques auxquelles nous nous sommes intéressés paraissent fortement discriminantes. Ce phénomène concerne aussi bien les sources de revenu que le montant du revenu, la disponibilité d'un compte dans une institution financière formelle, voire le dépôt informel de monnaie.

\subsubsection{Tontine et sources de revenus}

Une question avait pour but de déterminer la source principale de revenu et de voir si la population qui participe à des tontines dispose de revenus de provenance différente de ceux dont dispose la population non tontineuse. Quelques personnes ont fait montre d'une certaine réticence à indiquer la source de leurs revenus. Il est remarquable de constater qu'il s'agit uniquement de personnes qui ne pratiquent pas la tontine ; ceci peut introduire des biais importants et les conclusions de ce paragraphe et du suivant pourraient en être affectées. Dans ces conditions, les résultats suivants ont été observés :

TABleAU 2

Source principale de revenu et participation à une tontine

\begin{tabular}{lrcc}
\hline $\begin{array}{l}\text { Pratique la tontine } \\
\text { Source de revenu }\end{array}$ & Oui & Non & Total \\
\hline Traitements et salaires & 29 & 47 & 76 \\
Pension & 3 & 10 & 13 \\
Revenus de l'entreprise & 16 & 16 & 32 \\
Revenus de l'agriculture & 0 & 11 & 11 \\
Revenus de l'élevage & 4 & 5 & 9 \\
Autres & 6 & 17 & 23 \\
\hline
\end{tabular}


Sur les 58 personnes à tontiner dans notre échantillon, 29 sont des salariés : dans $50 \%$ des cas, le tontineur est un salarié ; dans plus de $27 \%$ des cas, il s'agit d'un entrepreneur. On note toutefois que si les salariés sont, en proportion, les plus nombreux à tontiner, ils ne constituent pas un groupe qui présente une vocation spéciale à tontiner : la proportion de salariés qui tontine ne diffère pas significativement de celle qui ne tontine pas. Il n'en est pas de même des entrepreneurs qui tontinent sensiblement plus que les autres groupes de titulaires de revenus.

Un test du $\chi^{2}$ indique des sources de revenu différentes entre tontineurs et non-tontineurs; bien que ce résultat ne soit que très légèrement significatifi ${ }^{12}$, ceux qui tontinent disposent plus fréquemment de revenus d'entreprises et, très nettement, beaucoup moins souvent de revenus de l'agriculture. Les tontineurs constituent une population intégrée dans les circuits de la modernité et qui a abandonné les vestiges urbains du mode de vie rural (agriculture périurbaine ou interstitielle dans le tissu urbain).

\subsubsection{Revenus et pratique de la tontine}

Le dépouillement de tous les questionnaires a abouti au résultat qu'un certain nombre d'individus survivent à Niamey sans revenu : on compte ainsi 33 personnes, toutes non tontineuses. Parmi les personnes non tontineuses le revenu nul constitue le revenu modal. Quoique étonnant, le chiffre de $17 \%$ des individus sans revenus ne paraît pas impossible : on sait les difficultés de la vie et de la survie dans les villes africaines et l'on peut penser que la survie de ces personnes passe par la mendicité ou est mise sur le compte de la solidarité à l'intérieur des familles ou des ménages. Une très forte différenciation apparaît concernant le montant du revenu entre les tontineurs et les non-tontineurs, ce dont témoigne le tableau suivant :

TABLEAU 3

Revenu mensuel et pratique de la tontine

\begin{tabular}{lccc}
\hline $\begin{array}{l}\text { Pratique la tontine } \\
\text { Revenu mensuel (FCFA) }\end{array}$ & Oui & Non & Total \\
\hline $0 \mathrm{~F}$ & 0 & 33 & 33 \\
$10,000 \mathrm{~F}$ et moins & 2 & 12 & 14 \\
Entre 10000 et $20000 \mathrm{~F}$ & 3 & 18 & 21 \\
Entre 20000 et $40000 \mathrm{~F}$ & 10 & 20 & 30 \\
Entre 40 000 et 60 000 F & 23 & 26 & 49 \\
Plus de 60 000 F & 20 & 26 & 46 \\
Total & 58 & 135 & 193 \\
\hline
\end{tabular}

12. Le risque de première espèce est de $4 \%$. 
Un test de $\chi^{2}$ indique une différence de revenu entre les tontineurs et les non-tontineurs significative à 1 pour 10000 ! La conclusion est claire : les personnes qui participent à une tontine disposent de revenus plus élevés que les autres.

Cette conclusion est susceptible d'apparaître un peu forcée parce que l'on a inclu parmi ceux qui ne pratiquent pas la tontine de nombreuses personnes à revenus nuls. Si nous écartons ces personnes, la discrimination subsiste (quoique évidemment moins fortement). Ainsi, même en ne tenant pas compte de l'ensemble des enquêtés à revenus nuls, la personne qui participe à une tontine dispose de plus de revenus que la moyenne.

\subsubsection{Institution financière formelle et pratique de la tontine}

Nous venons de constater que les personnes qui se livrent à la pratique tontinière disposent toujours de revenus et que ces revenus sont en moyenne plus élevés que ceux des individus qui ne tontinent pas.

On pourrait s'attendre à ce que les personnes qui pratiquent la tontine ne s'intéressent pas aux institutions financières formelles et que le monde du formel soit fermé de fait à ceux qui se préoccupent d'abord de l'informel. Il n'en est rien, bien au contraire, et il s'agit là d'un des points les plus remarquables de cette enquête. Si l'on considère que disposer d'un compte en banque, d'un compte courant postal ou d'un compte à la Caisse Nationale d'Épargne (CNE) signifie que l'on s'adresse à la finance formelle, alors les tontineurs se tournent plus volontiers vers les organismes financiers formels que les non-tontineurs. Une forte différenciation des comportements apparaît : plus des deux tiers des tontineurs disposent d'un compte dans une institution formelle alors que $38 \%$ seulement des non-tontineurs sont dans le même cas. La différence de comportement est particulièrement significative (à plus de 1 pour 10 000). Poussons notre investigation et distinguons les hommes des femmes. Les données sont les suivantes :

\section{TABLEAU 4}

Possession d'un compte dans une institution financière formelle et pratique de la tontine, par sexe

\begin{tabular}{lccccccc}
\hline Sexe & \multicolumn{5}{c}{ Masculin } & \multicolumn{3}{c}{ Féminin } & \\
\cline { 2 - 7 } $\begin{array}{l}\text { Possède un compte } \\
\text { Participe à une tontine }\end{array}$ & Oui & Non & Total & Oui & Non & Total & $\begin{array}{c}\text { Total } \\
\text { général }\end{array}$ \\
\hline Oui & 17 & 4 & 21 & 23 & 14 & 37 & 58 \\
Non & 36 & 47 & 83 & 16 & 36 & 52 & 135 \\
Total & 51 & 53 & 104 & 39 & 50 & 89 & 193 \\
\hline
\end{tabular}


Parmi les hommes, plus de $80 \%$ des tontineurs possèdent un compte dans une institution formelle contre seulement $43 \%$ parmi les non-tontineurs ${ }^{13}$. Parmi les femmes, le même type de déséquilibre est noté : presque les deux tiers des tontineuses possèdent un compte dans une institution formelle contre moins d'un tiers parmi les non-tontineuses ${ }^{14}$. Ainsi, tant la femme que l'homme qui participe à une tontine est tourné vers le monde financier formel.

\subsubsection{Tontine et dépôt chez un garde-monnaie}

Près de $14 \%$ des tontineurs enquêtés déposent des fonds chez un garde-monnaie alors que seuls $2 \%$ des non-tontineurs agissent de même. Cette différence est particulièrement significative ${ }^{15}$ quand on considère l'ensemble des enquêtés.

Mais notre intérêt est encore plus éveillé par l'hétérogénéité des comportements entre hommes et femmes. Les hommes se tournent peu vers les gardesmonnaie (au point qu'il n'existe pas de différence significative entre hommes tontineurs et non tontineurs), alors que les femmes qui pratiquent la tontine ont systématiquement plus recours aux gardes-monnaie que celles qui ne tontinent pas. Ces différences de comportement ressortent clairement du tableau ci-après :

\section{TABLEAU 5}

Dépôt d'argent chez un garde-monnaie et pratique de la tontine, par sexe

\begin{tabular}{|c|c|c|c|c|c|c|c|}
\hline \multirow{2}{*}{$\begin{array}{l}\text { Sexe } \\
\text { Dépose chez un } \\
\text { garde monnaie } \\
\text { Participe à une tontine }\end{array}$} & \multicolumn{3}{|c|}{ Masculin } & \multicolumn{3}{|c|}{ Féminin } & \multirow{2}{*}{$\begin{array}{l}\text { Total } \\
\text { général }\end{array}$} \\
\hline & Oui & Non & TOTAL & Oui & Non & TOTAL & \\
\hline Oui & 1 & 20 & 21 & 7 & 30 & 37 & 58 \\
\hline Non & 1 & 82 & 83 & 2 & 50 & 52 & 135 \\
\hline Total & 2 & 102 & 104 & 9 & 80 & 89 & 193 \\
\hline
\end{tabular}

Ainsi, il apparaît que si la femme qui tontine se tourne plus que celle qui ne tontine pas vers le monde financier formel, elle s'adresse aussi plus que cette dernière au monde financier informel. Son comportement apparaît comme le résultat d'un choix délibéré en direction des deux types de gestion du portefeuille; le comportement de l'homme est moins tranché.

Le portrait du tontineur se trouve alors largement amendé par ces éléments de différenciation économique.

13. La différence de comportement est significative à $2 \%$ environ.

14. La différence de comportement est significative à $3,3 \%$ environ.

15. Le risque de première espèce est de $1,5 \%$. 


\section{Le tontineur}

En montrant ce qui distingue le tontineur du non-tontineur, nous avons esquissé indirectement les caractéristiques du tontineur: tout d'abord, il s'agit d'une femme ( $2 / 3$ des cas); son revenu provient du salaire (50\% des cas) ou de l'entreprise ( $27 \%$ des cas) ; s'il s'agit d'une femme, c'est surtout entre 31 et 40 ans qu'elle participe à une tontine. Bien sûr, le tontineur a un revenu largement supérieur à celui du non-tontineur; il s'adresse plus fréquemment que ce dernier au monde financier formel, mais aussi, si c'est une femme, au garde-monnaie. Le portrait établi jusqu'à présent est surtout différentiel, en ce sens que nous cherchions les traits qui différenciaient le tontineur du non-tontineur.

Une approche plus positive s'impose maintenant pour affiner le portrait du tontineur dont nous connaissons déjà les caractéristiques générales. En particulier, nous allons nous intéresser à la manière dont il gère son argent, entre les différentes possibilités qui lui sont offertes.

\subsection{Les finances du tontineur}

Participer à une tontine ressort typiquement de l'activité informelle. Il en est de même du dépôt d'argent chez un garde-monnaie. Mais le tontineur ne se cantonne pas au monde informel : il s'adresse aussi au monde formel : ainsi, près d'un tontineur sur deux dispose d'un compte en banque, plus d'un sur deux d'un compte à la Caisse Nationale d'Épargne (CNE). Globalement, $68 \%$ des tontineurs disposent d'au moins un compte dans une institution formelle et une étude plus fine montre que $35 \%$ des tontineurs s'adressent à deux institutions formelles. Détaillons ces résultats globaux.

\subsubsection{La disposition d'un compte en banque}

L'ouverture d'un compte dans une banque demande une très grosse immobilisation de capital et le paiement de frais de gestion considérés comme particulièrement élevés ${ }^{16}$. Pourtant, $45,6 \%$ des tontineurs disposent d'un compte bancaire ${ }^{17}$. La différenciation sexuelle, pour importante qu'elle paraisse au dépouillement,

16. À titre d'exemple, et à comparer avec le salaire minimum garanti à Niamey qui s'élève à 18898 FCFA, le dépôt minimum d'ouverture d'un compte chèque à la BCCI est de $500000 \mathrm{~F}$ et les frais de gestion du compte sont de $3510 \mathrm{~F}$ par trimestre.

17. Il est possible que cette proportion inclue des déclarations de personnes qui, en fait, partagent un compte pour percevoir des rémunérations. 
n'est pas statistiquement significative au seuil de $5 \%$ : il nous semble toutefois probable que les femmes qui tontinent disposent moins fréquemment de comptes bancaires que les hommes, mais ceci n'est pas prouvé.

TABLEAU 6

Disposition d'un compte bancaire selon le sexe parmi les tontineurs

\begin{tabular}{lccc}
\hline Sexe & & & \\
Dispose d'un compte bancaire & Masculin & Féminin & Total \\
\hline Oui & 12 & 14 & 26 \\
Non & 8 & 23 & 31 \\
Total & 20 & 37 & 57 \\
\hline
\end{tabular}

Si la différenciation sexuelle n'est pas prouvée dans la disposition d'un compte bancaire, il n'en est pas de même des sources de revenu.

Il était attendu que les salariés disposent plus fréquemment de comptes bancaires que les autres titulaires de revenus. En effet, et ceci est surtout valable dans la fonction publique, pallier l'absence d'un compte bancaire entraîne de lourdes démarches lors de la paye : pour les éviter, un fonctionnaire sera souvent prêt à payer le prix demandé par les banques. Le résultat obtenu n'est donc pas étonnant :

TABLEAU 7

Disposition d'un compte bancaire et revenu salarial parmi les tontineurs

\begin{tabular}{lccc}
\hline $\begin{array}{l}\text { Type de revenus } \\
\text { Dispose d'un compte bancaire }\end{array}$ & $\begin{array}{c}\text { Traitements } \\
\text { et salaires }\end{array}$ & Autres & Total \\
\hline Oui & 20 & 6 & 26 \\
Non & 9 & 22 & 31 \\
Total & 29 & 28 & 57 \\
\hline
\end{tabular}

Les tontineurs salariés disposent plus fréquemment de comptes bancaires que les autres titulaires de revenus. Notons que parmi les salariés, ceux qui disposent d'un compte bancaire et ceux qui n'en disposent pas gagnent, à quelques francs près, le même salaire moyen. Pour les salariés, ce n'est pas le montant du salaire qui est la raison de la disposition d'un compte bancaire. Réciproquement, pour les titulaires de revenus non salariaux, ceux qui disposent d'un compte bancaire ont un revenu près de 4 fois supérieur à celui de 
ceux qui n'en disposent pas ${ }^{18}$. Pour les non-salariés, un montant élevé de revenu induit la disponibilité d'un compte bancaire. Par contre, les non-salariés qui ne disposent pas d'un compte en banque n'en trouvent pas la raison dans la modicité de leurs revenus : ceux-ci sont du même ordre de grandeur que ceux des salariés ${ }^{19}$. La non-disponibilité d'un compte bancaire semble, dans le cas des non-salariés modestes, plus liée à une question de statut social ou professionnel qu'au niveau du revenu.

Nous nous attendions au même type de résultat de la part des titulaires de revenus d'entreprise (commerçants, artisans,... ). Il n'en a rien été et l'on découvre que ces derniers disposent significativement de moins de comptes en banque que les autres.

TABLEAU 8

Disposition d'un compte bancaire et revenu de l'entreprise

\begin{tabular}{lccc}
\hline $\begin{array}{l}\text { Type de revenus } \\
\text { Dispose d'un compte bancaire }\end{array}$ & $\begin{array}{l}\text { Revenus de } \\
\text { l'entreprise }\end{array}$ & Autres & Total \\
\hline Oui & 4 & 22 & 26 \\
Non & 12 & 19 & 31 \\
Total & 16 & 41 & 57 \\
\hline
\end{tabular}

L'explication de ce comportement semble être la suivante : la plupart des revenus d'entreprise sont relativement réduits parmi les non-détenteurs de compte en banque (moyenne des déclarations : $66080 \mathrm{~F}, s=61180 \mathrm{~F}, n=12$ ). À l'inverse, les détenteurs de comptes, peu nombreux, déclarent toucher de gros revenus (moyenne : $263750 \mathrm{~F}, s=163570 \mathrm{~F}, n=4$ ).

Nous retrouvons la logique économique : les gens ayant les revenus les plus élevés disposent de plus de comptes bancaires que les plus pauvres; ils peuvent les financer d'une part, et y trouvent des avantages, d'autre part. Les coûts d'un compte bancaire dépassent les avantages pour un petit entrepreneur, alors qu'il en va inversement pour un grand.

18. 203170 FCFA contre 52600 FCFA.

19. 52600 FCFA (avec $s=48150 \mathrm{~F}$ et $n=22$ ) contre les chiffres cités plus haut. 


\section{Conclusion}

Parmi les tontineurs, s'adressent à la banque les salariés et les entrepreneurs les plus importants; ne s'y adressent pas les petits entrepreneurs. Les femmes, quoique se tournant moins vers les banques que les hommes de notre échantillon, ne peuvent, statistiquement parlant, être considérées comme un groupe particulier à l'égard des banques. S'adresser à une banque pour y ouvrir un compte apparaît comme une démarche volontariste qui n'est pas à la portée du plus grand nombre. De plus, le revenu n'est pas le seul déterminant de cette démarche ; le sexe et le statut social ou professionnel semblent aussi jouer un rôle important.

\subsubsection{Le compte à la Caisse Nationale d'Épargne}

La Caisse Nationale d'Épargne a la volonté d'être un instrument d'épargne populaire : le solde minimum du livret à l'ouverture n'est que de 2000 FCFA, la commission de tenue de compte n'existe pas, le compte rapporte un intérêt de $6,5 \%$ par an sur le solde calculé deux fois par mois; enfin, pour limiter les retraits, la Caisse Nationale d'Épargne a institué une commission de retrait de $200 \mathrm{~F}$.

Sur les 57 tontineurs de notre échantillon, 32, soit $56 \%$ de l'effectif, disposent d'un livret à la CNE. Incontestablement, la CNE est populaire parmi les tontineurs. En fait, elle est tellement populaire qu'aucune des caractéristiques de ceux qui possèdent un livret ne diffère significativement de celle des tontineurs qui n'en ont pas :

- Il n'existe pas de différence entre la proportion des tontineurs hommes ou femmes qui disposent d'un livret à la $\mathrm{CNE}$; de plus, les revenus moyens des hommes ayant un livret à la CNE ne se distinguent pas de ceux des femmes qui y disposent d'un livret; enfin, ces revenus ne diffèrent pas des revenus de ceux qui n'ont pas de livrets.

- Ni les salariés, ni les titulaires de rcvenus de l'entreprise ne se distinguent des autres titulaires de revenus en ce qui concerne la disposition d'un compte à la CNE.

\section{Conclusion}

La CNE a la volonté d'être une institution financière formelle populaire, à la portée de chaque individu. Dans ce sens, on peut affirmer que la CNE a réussi sa mission : quand un individu désire épargner, elle est à sa disposition. Réciproquement, la facilité d'ouverture d'un compte à la CNE conduit à une 
clientèle non spécifique, qui ne se distingue pratiquement pas de la nonclientèle : la CNE n'a pas en face d'elle des groupes cibles comme les banques (salariés, commerçants importants,...), mais une masse indifférenciée de clients potentiels.

Ce point est largement confirmé quand on étudie la disponibilité conjointe d'un compte dans une banque et d'un livret à la CNE. On compte 19 individus sur les 57 étudiés (soit $33 \%$ des cas) qui disposent à la fois d'un compte dans une banque et d'un livret à la CNE. On note que ces 19 individus représentent $73 \%$ des 26 personnes ayant un compte dans une banque et $59 \%$ des 32 personnes qui possèdent un livret à la CNE. Il est remarquable de constater que le revenu moyen des titulaires d'un compte en banque ne se distingue pas de celui des individus qui disposent à la fois d'un compte en banque et d'un livret à la CNE. La sélectivité introduite par les banques suffit comme critère distinctif : la prise en compte de la disposition d'un livret à la CNE n'apporte rien.

\subsubsection{Les finances informelles du tontineur}

Celui qui tontine agit dans le monde informel. Mais d'autres solutions à ses problèmes financiers existent dans le monde informel, en particulier les gardesmonnaie.

Sur les 57 personnes de notre échantillon, 8 (c'est-à-dire $14 \%$ de l'effectif observé) s'adressent à des gardes-monnaie. Certes, il s'agit de sept femmes et de un homme, mais cette distinction n'est pas statistiquement significative, pas plus que celle concernant les sources de revenus. Seul un échantillon substantiellement plus large aurait pu nous permettre de découvrir les éléments de segmentation caractérisant les clients des gardes-monnaie. Notons enfin que deux personnes s'adressent aussi pour leurs finances à des caisses de la Samaria ${ }^{20}$ de leur quartier.

\subsection{La participation multiple à des tontines}

Pour le moment, nous nous sommes intéressés aux tontineurs en général, mais la réalité est susceptible d'être plus complexe. Aussi, notre questionnaire en a-t-il tenu compte ${ }^{21}$.

On sait que la participation à une tontine aide à gérer tant l'épargne que l'encaisse. Mais la participation à une tontine limite parfois la souplesse de la

20. Samaria : association traditionnelle de jeunesse, reconstituée pour servir de structure de mobilisation par et pour le parti au pouvoir.

21. En partie seulement, comme on le verra plus loin. 
gestion qui est désirée ; d'autre part, cette participation peut, dans certains cas, se révéler nécessaire pour tisser des liens utiles à la vie sociale. Dans ces conditions, un individu peut être conduit à participer à plusieurs tontines. En général, on pense qu'il s'agit d'un comportement relativement peu fréquent, mais qui existe. C'est dans ce cadre que nous avons tenté d'évaluer la participation d'un tontineur à plusieurs tontines. Les résultats trouvés à Niamey sont surprenants :

TABlEAU 9

Participation à plusieurs tontines

\begin{tabular}{lcc}
\hline Participe à d'autres tontines & Nombre d'observations & Fréquence relative ( \%) \\
\hline Oui & 12 & 21 \\
Non & 45 & 79 \\
Total & 57 & 100 \\
\hline
\end{tabular}

Sur les 57 personnes interrogées, 12 participent à d'autres tontines que celle sur laquelle ils ont donné les précisions précédentes. Ainsi, dans $21 \%$ des cas, on ne tontine pas seulement une fois, mais plusieurs : on aime tontiner et la tontine fait partie de la vie. Cette prévalence de participations multiples à des tontines est particulièrement importante, puisqu'un cinquième des tontineurs appartient à deux tontines au moins. La participation à plus d'une tontine n'est pas un phénomène inhabituel, mais au contraire un comportement fréquent à Niamey.

La conséquence en est la suivante : comme notre enquête a été effectuée sur des individus, la véritable prévalence de la tontine en milieu urbain doit être réévaluée d'un cinquième, étant entendu que cette dernière proportion concerne les tontines multiples auxquelles participe un individu et non une correction concernant une proportion d'individus dans la population qui participent à une tontine.

Il est alors intéressant de voir les caractéristiques du « tontineur multiple ». Tout d'abord, on note que les principaux éléments de segmentation ne jouent pas dans cette occurrence. Ainsi, aucune différence significative ne peut être mise en évidence entre sexes : en moyenne, le comportement des hommes et des femmes est le même en ce qui concerne la participation à d'autres tontines. De même, celui qui participe à plusieurs tontines ne fait pas de versements plus importants que les autres tontineurs. Enfin, sa source de revenu est généralement le salaire ou le revenu de l'entreprise, mais statistiquement, la source de revenu n'est pas significative. Plus intéressant est le niveau du revenu que déclare le «tontineur multiple ». Alors que le revenu moyen déclaré est de 
$71910 \mathrm{~F}$, celui du « tontineur multiple » est de $124040 \mathrm{~F}$ (avec $s=134310 \mathrm{~F}$ ce qui dénote un important étalement à droite - et $n=12$ ) : il lui est donc significativement supérieur. On ne sera pas étonné de constater que ce « tontineur multiple » dispose plus fréquemment d'un compte en banque que le tontineur simple. Notons qu'une fois de plus, les «tontineurs multiples » ne disposent pas d'une proportion significativement différente de livrets à la Caisse Nationale d'Épargne, ce qui confirme nos déductions précédentes sur la nonspécificité de la clientèle de la CNE.

\section{Conclusion}

Le «tontineur multiple » est un personnage plus aisé que la moyenne. De ce fait, il dispose habituellement d'un compte en banque (dans $83 \%$ des cas) et d'un livret à la CNE (75 \% des cas, mais ceci ne le distingue pas des autres tontineurs). Comme le «tontineur multiple » tontine au moins deux fois, on peut dire qu'il dispose généralement de 4 actifs financiers. Dans cette optique, la tontine est une modalité spécifique de gestion d'actif qui est particulièrement appréciée par un individu profondément intégré au monde moderne.

\section{Conclusion}

\section{Le portrait du tontineur de Niamey}

Participer à une tontine est une pratique ancienne en Afrique : aussi loin que remonte la mémoire, on y connaît des tontines. Les tontines appartiennent au monde ancien de l'Afrique et ressortent de comportements et d'habitudes profondément ancrées dans les civilisations locales; la participation aux tontines serait le signe de la stabilité de ces sociétés ${ }^{22}$.

On pourrait donc penser que le tontineur urbain de la fin du $\mathrm{xx}^{\mathrm{e}}$ siècle est un homme du passé qui a des difficultés à quitter le monde rural et qui reste cramponné à des formes d'épargne dépassées et inadaptées. On s'attendrait à un monde tontinier replié sur lui-même, tournant dans les mêmes cercles, ne s'adressant pas au monde financier formel : le tontineur est supposé ignorer le système financier formel et ce dernier est jugé incapable de fournir une prestation utile au tontineur. D'après ce portrait, la participation à une tontine apparaî-

22. À la limite, on peut considérer la tontine comme un système de dons et contre-dons, étendu à un groupe et équilibré sur une période. 
trait comme un comportement résiduel, voué à la disparition au profit de formes plus modernes de gestion financière ${ }^{23}$.

Le portrait du tontineur, tel qu'il ressort de notre enquête, est à l'opposé de cette image, au point que cette dernière décrit le contraire de la réalité. Tout d'abord, il convient de noter que le tontineur possède des revenus, ce qui est loin d'être toujours le cas des non-tontineurs. En second lieu, les sources de revenu du tontineur sont modernes. La source de ses revenus est caractéristique : le tontineur perçoit un salaire ou des revenus de son activité entrepreneuriale. Par contre, il ne dispose pas comme le non-tontineur de revenus de l'agriculture périurbaine : le non-tontineur fait l'effet d'être un individu laissé de côté dans le développement, dont le comportement résiduel serait voué à la disparition; le tontineur par son activité semble appartenir au monde de l'avenir et de la modernité. D'autre part, le montant des revenus est plus élevé parmi les tontineurs que parmi les autres personnes : le tontineur est un individu plus à l'aise que la moyenne, son revenu est de $27 \%$ supérieur à celui du non-tontineur qui dispose d'un revenu et de $60 \%$ supérieur à celui de l'ensemble des non-tontineurs.

Le tontineur, cet homme riche, ayant des revenus de source moderne, $s$ 'adresse pour sa gestion financière beaucoup plus aux institutions financières formelles que le non-tontineur. Nous sommes loin de l'idée que l'on participe à une tontine parce que l'on ne connaît pas les banques ou que l'on n'a pas accès au système financier formel. Le tontineur connaît bien les avantages que peuvent lui apporter les banques et il y recourt. Son originalité consiste à ne pas se limiter à une seule forme d'intermédiation financière ${ }^{24}$ mais, précisément, à s'adresser aux deux mondes. Son comportement en ce qui concerne les questions d'argent semble être celui d'un choix délibéré de faire appel aux formes

23. À l'époque où le développement était vu de façon linéaire, la tontine apparaissait comme une forme résiduelle et vouée à la disparition. Deux arguments la condamnaient :

- Dans une coupe instantanée, la tontine est moins utilisée par les riches que par les pauvres: " $\mathrm{La}$ tontine est moins populaire chez les évolués que chez les travailleurs » (L. Baeck, 1961).

- Les études chronologiques constatent la régression de la pratique tontinière : ainsi, à Bukavu, un inspecteur du travail note à propos des tontines : "Cette coutume semble d'ailleurs non seulement se simplifier, perdre de sa solennité et de son formalisme, mais aussi avoir parfois tendance à disparaître » (J. Ghilain, 1968).

Notons qu'au terme de notre étude, nous ne saurions exclure aucune de ces hypothèses, les évolutions pouvant être cachées par la régression des revenus, la déconfiture du système financier moderne, etc.

24. On peut en effet considérer la tontine comme une forme fermée sur elle-même et rudimentaire d'intermédiation financière dans laquelle les premiers à lever sont emprunteurs nets et les demiers, prêteurs nets. 
financières formelles et aux formes financières traditionnelles : parce qu'il est riche, le tontineur, pour gérer son encaisse, utilise les banques, la Caisse Nationale d'Épargne, ainsi que les tontines et les gardes-monnaie. Puisqu'il utilise toutes ces formes, on peut penser qu'il répartit son encaisse en utilisant les avantages de chaque institution. La tontine ne s'oppose pas aux institutions financières formelles, elle les complète et offre ainsi une autre pratique de gestion du portefeuille à l'individu.

Un dernier mot : très fréquemment, ce tontineur moderne est... une « tontineuse ». Ce point est d'autant plus appréciable que nous sommes dans un pays profondément musulman, et que fréquemment, on laisse entendre que dans de tels cas, les femmes sont à la traîne de la modernité et restent, plus que les hommes, engluées dans le passé. La femme qui participe à une tontine constitue très nettement une force importante de changement dans la société nigérienne : c'est une femme qui s'émancipe, qui acquiert son indépendance financière. Fréquemment, elle dispose d'un compte dans une institution financière formelle. Sa participation à une tontine est le signe de bouleversements en marche.

En conclusion, le tontineur apparaît comme une personne au comportement moderne, profondément intégrée dans la société, agissant dans le monde en disposant de plus d'informations que le commun des individus et constituant une force de mouvement dans la société.

\section{Bibliographie}

BAECK, L. (1961), "An expenditure study of the congolese evolues of Leopoldville, Belgian Congo » dans Social Change in Modern Africa, p. 159-181, Oxford University Press for the International African Institute, Londres.

BAscom, W.R. (1952), «The Esusu : a credit institution of the Yoruba », The Journal of the Royal Anthropological Institute of Great Britain and Ireland, vol. 82, part. I.

Baulier, F, A. Corrèze, A. Lebissonnais et C. Ostyn (1988), Les tontines en Afrique, Paris, Caisse centrale de coopération économique, Notes et Etudes, $n^{\circ} 12$, septembre.

Bouman, F.J.A. (1977), « Indigenious saving and credit societies in the Third World : a message », Saving and Development, vol. I, $\mathrm{n}^{\circ} 4$.

Bouman, F.J.A. (1979) «The ROSCA : financial technology of informal savings and credit institutions in developing countries ", Saving and Development, vol. III, $n^{\circ} 4$. 
Bouman, F.J.A. et K. Harteveld (1976), «The Djanggi : a traditional form of saving and credit in West Cameroon ", Sociologica ruralis, vol. XVI, $\mathrm{n}^{\circ}$ 21-2.

Cissé, D. (1969), Les problèmes de la formation de l'épargne en Afrique, Paris, Présence africaine.

Delancey, M. (1977), "Credit for the common man », Journal of Modern African Studies, vol. XV, $\mathrm{n}^{\circ} 2$.

DiarRa, M.F. (1984), Étude de factibilité pour la mise en place des caisses d'épargne villageoises, Niamey, IRSH,juin.

GeERTZ, C. (1961), « The rotating credit association : a "middle rung" in development », Economic Development and Cultural Change, vol. $\mathrm{X}, \mathrm{n}^{\circ} 1$, octobre.

GhIlAIN, J. (1968), « À propos de la formation du capital autochtone en Afrique noire : le Likelemba ", Revue de l'institut de sociologie, $n^{\circ} 2$, Université libre de Bruxelles.

Graham, D., C. Cuevas, K. Negash, M. Keita et M. Masini (1987), Finances rurales au Niger: une évaluation critique et des propositions de réforme, Ohio State University - USAID, Niamey, février, p. 182, 235, 238.

Graham, D., C. Cuevas, K. Negash, M. Keita et M. Masini (1986), Financial Markets in Rural Niger : Formal and Informal Transactions on the Household Level, Ohio State University Colombus, mars.

HAGGBLADE, S. (1978), « Africanization from below : the evolution of cameroonian saving societies into western-style banks », Rural Africana, $\mathrm{n}^{\circ} 2$.

Harteveld, K. (1982), Saving and Credit in the Grassfields, Manuscrit non publié, Pays-Bas, Université de Wageningen.

HILl, C. (1977), Agricultural Credit Assessment : Cameroon, Douala, USAID.

IвRо, C. (1987) « Tontines de femmes », l'Opérateur économique, $\mathrm{n}^{\circ} 4$, Niamey, novembredécembre.

Kamwa, P. (1985), « Monétarisation et tontines dans le financement du processus de développement : l'exemple du Cameroun », Thèse de $3^{\mathrm{e}}$ cycle, Orléans.

KESSLER, D. et P.A. Ulumo (1985) (ouvrage collectif publié sous la direction de), Épargne et développement, Paris, Economica.

LelarT, M. (1984), «L'épargne informelle en Afrique », Communication au Colloque du Caire : «Les politiques financières nationales et la formation du capital en Afrique », Le Caire, juillet.

LeLART, M. (1988), L'épargne informelle en Afrique. Essai de typologie des tontines, Orléans, Institut orléanais de finances, juin.

LELART, M. (1988), « Les informalités financières : les tontines », Communication au Colloque de Nouakchott : "Les pratiques informelles comparées : les fondements de la non légalité », Orléans - Nouakchott, décembre. 
Liman TinguiRI, L. (1987), «Épargne et crédit informels ", l'Opérateur économique, $\mathrm{n}^{\circ} 4$, Niamey, novembre-décembre.

Liman Tinguiri, L. (1988), Étude d'identification pour un projet de développement de l'artisanat du cuir, Niamey.

Liman Tinguiri, L. et C. Rietsch (1989) Protocole d'enquête sur les tontines en milieu urbain à Niamey, Niamey, UREF - Université de Niamey, juin.

Massing, A. et D. Selbel (1974), Traditional Organisations and Economic Development : Studies of Indigenous Cooperatives in Liberia, New York, Praeger Publishers.

Miller, L. et F. OKori, Esusu Clubs and Their Performances in Mobilizing Rural Saving and Credit, Department of Agricultural Economics, University of Ibadan, Ibadan.

Miracle, M.P., D.S. Miracle et L. Cohen (1980), « Informal savings mobilization in Africa », Economic Development and Cultural Change, vol. XXVIII, $n^{\circ} 24$.

Mourgues, N. (1985), « Les tontines. Analyse des mécanismes financiers », Table ronde sur les circuits informels et le financement des entreprises, Bangui, mai. Publié par l'Institut orléanais de finances, Orléans.

Nicolas, G., H. Magagi et M. DAN Mouché (1968), Le système traditionnel de crédit dans la région de Maradi, Mission d'études socio-économiques du pays Haoussa Oriental, CNRSH, Niamey.

Oudin, X. (1989), Diagnostic du secteur informel urbain au Niger, Niamey, février.

RIETSCH, C. (1990), «Les paramètres de base des tontines à Niamey », UREF, Notes de recherche, $\mathrm{n}^{\circ} 12$, Paris.

RIETSCH, C. (1990), La tontine dans les difficultés, Université de Niamey, juin.

RIETSCH, C. (1991), « La technique de la tontine », Annales de l'Université de Niamey, t. IV, Niamey.

SERVET, J.M. (1985), « Un système alternatif d'épargne et de crédit : les tontines africaines ", Reflets et perspectives de la vie économique, t. XXIV, $\mathrm{n}^{\circ} 1$.

WoILlET, J.C. (1989), Le financement de l'artisanat au Niger, Niamey, BIT - PNUD, décembre. 